\title{
Structural slow light can enhance Beer-Lambert absorption
}

\author{
Isabelle Dicaire, Sanghoon Chin, and Luc Thévenaz \\ Ecole Polytechnique Fédérale de Lausanne, Institute of Electrical Engineering, STI-GR-SCI-LT Station 11, CH-1015 Lausanne, Switzerland \\ isabelle.dicaire@epfl.ch, luc.thevenaz@epfl.ch
}

\begin{abstract}
We experimentally demonstrate that structural slow light can enhance Beer-Lambert absorption. A 4-fold reduction of the group velocity induced by mere cavity effects has caused an increase of molecular absorption by $130 \%$.

OCIS codes: (070.5753) Resonators; (060.2370) Fiber optics sensors; (060.5295) Photonic crystal fibers
\end{abstract}

Molecular absorption of gases is usually expressed by means of the Beer-Lambert law, stating that light transmitted though an absorbing medium will decay exponentially as $I=I_{0} \exp \left(-\alpha_{L} L\right)$, where $\alpha_{L}$ is the absorption coefficient and $L$ is the optical path length. Previous studies have suggested that the absorption coefficient is inversely proportional to the group velocity [1]. However we have obtained experimental results showing that there is no enhancement of molecular absorption using material slow light, where the microscopic response of the medium was modified using stimulated Brillouin scattering [2]. This experiment has been designed, so that the molecular absorption and the slow light generation are negligibly correlated and can be controlled independently without mutually interfering. In this paper we use the same experimental approach to show that the Beer-Lambert absorption of molecules can be enhanced with structural slow light. The structure of the slow light system is designed to be macroscopic using long fiber ring resonators, so that the cavity can be conveniently opened or closed to operate the system either in standard or slow light regime, leaving the absorbing fiber segment untouched. Closing the cavity results in delayed propagation and longer effective optical path lengths through light recirculation.

The fiber ring resonator system was built according to Fig. 1 (left). The cavity round-trip loss was continuously varied using a variable optical attenuator (VOA) to modify the cavity-induced group delay. To determine precisely its value, the output of the laser diode was sinusoidally modulated at $70 \mathrm{kHz}$ by an electro-optic Mach-Zehnder intensity modulator (EOM) and the phase of the sine wave measured after propagation through the cavity. The largest time delay achieved through the cavity was $\sim 700 \mathrm{~ns}$ for a loop time of $260 \mathrm{~ns}$, corresponding to a group velocity reduced to $27 \%$ of its nominal value. Absorption profiles were obtained by applying a voltage ramp signal to the laser diode combined with lock-in detection to acquire clean signals. Fig. 1 (right) shows the enhancement of the molecular absorption effect as a function of the group index, and clearly demonstrates its linear dependence.
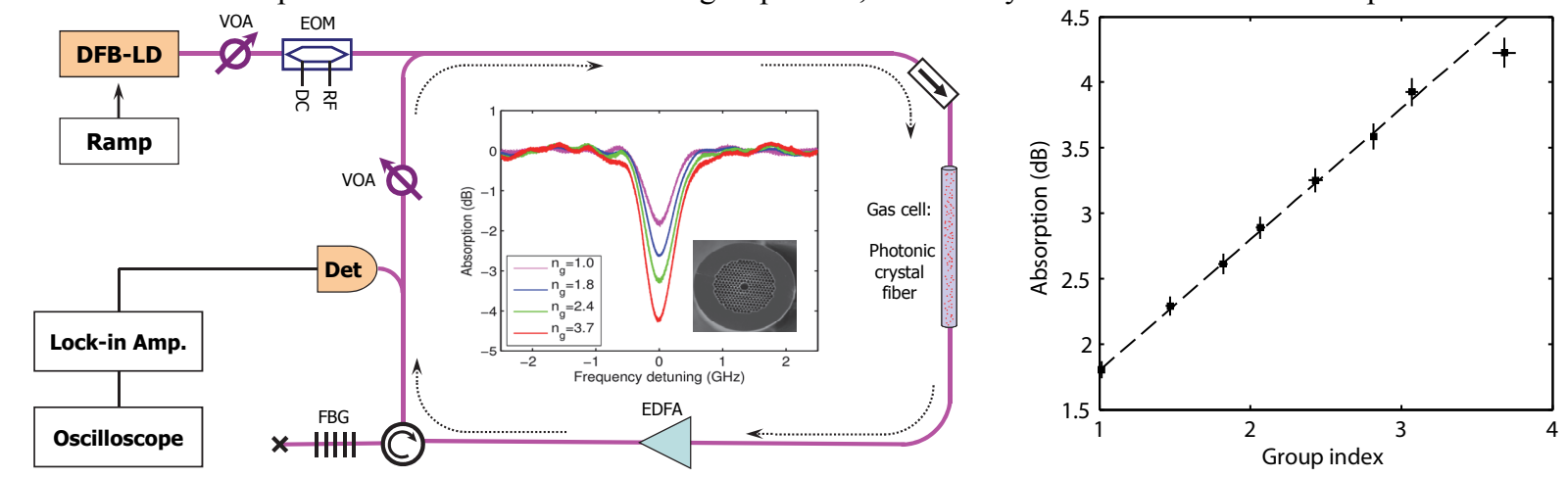

Figure 1, left: Experimental set-up realizing structural slow light using a fiber ring resonator system. Variable group delay is achieved by modifying the propagation loss in the fiber loop. The gas cell is made of a hollow-core photonic crystal fiber filled with acetylene gas and fusion spliced to standard single-mode fibers. Inset: Absorption profile as a function of the frequency detuning for several group delays. Right: Absorption of acetylene gas inside a hollow-core photonic crystal fiber as a function of the group index. The dashed line represents a linear fit with a slope equal to 1 on the semi-log graph, demonstrating the linear dependence of the absorption coefficient on the group index.

We have experimentally clarified that structural slow light can actually enhance the effect of Beer-Lambert absorption by molecules. Since different concepts of slow light systems give radically different responses, this highly suggests that the group index is not the appropriate universal quantity scaling the effective light-atom interaction strength, intuitively more closely related to an actual enhancement of the light energy density [3].

[1] N. A. Mortensen and S. Xiao, "Slow-light enhancement of Beer-Lambert-Bouguer absorption,” Appl. Phys. Lett. 90, 141108 (2007).

[2] S. Chin et al., "Material Slow Light Does Not Enhance Beer-Lambert Absorption," Slow \& Fast Light, OSA Technical Digest, SMA3 (2009).

[3] M.Santagiustina, "Electromagnetic energy velocity in slow light", Slow and Fast Light, OSA Technical Digest, SLTuD5 (2011). 